\title{
Validation of the 8th Edition of the UICC/AJCC Staging System for Nasopharyngeal Carcinoma From Endemic Areas in the Intensity-Modulated Radiotherapy Era
}

\author{
Ling-Long Tang, MDa,,; Yu-Pei Chen, MD ${ }^{\mathrm{a}, *}$; Yan-Ping Mao, MDa,b, $;$ Zi-Xian Wang, MD ${ }^{\mathrm{c},}$; Rui Guo, MD;

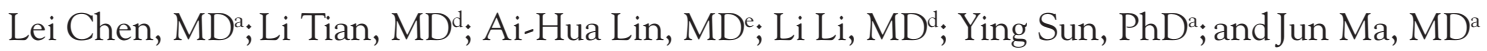

\begin{abstract}
Background: In this study, we evaluated the 8th edition of the Union for International Cancer Control (UICC)/AJCC staging system for nasopharyngeal carcinoma (NPC) in an endemic area, with the aim of validating its applicability and providing further information for future refinements. Methods: A total of 1,790 patients with newly diagnosed, non-distant metastatic, histologically proven NPC treated with intensity-modulated radiotherapy (IMRT) were retrospectively reviewed. The performance of various staging systems was compared using the Akaike information criterion (AIC) and Harrell's concordance index (c-index). Results: For N (node) category, the survival curves of different groups according to the 8th edition were well-separated, and the prognostic model predicted outcomes fairly well. The 8th edition had higher AIC and c-index values for all end points than the 7th edition. However, probably due to the improved locoregional control provided by IMRT, the survival curves for T2 and T3 almost overlapped, without significant differences in locoregional failure-free survival $(P=.606)$ and disease-free survival $(P=.735)$. Due to the difficultly of differentiating T2 and T3, the AIC and c-index values were similar for the T categories of the 7th and 8th editions. Similarly, the overall survival and disease-free survival curves for stage II and III disease were not clearly separated for either the 8th or 7th editions. Conclusions: The 8th edition of the UICC/AJCC staging system for NPC enables more accurate prediction of treatment outcomes. However, several limitations need to be addressed in future editions, and it would be reasonable to further optimize the T category classification.
\end{abstract}

From the aDepartment of Radiation Oncology, Sun Yat-sen University Cancer Center, State Key Laboratory of Oncology in South China, Collaborative Innovation Center for Cancer Medicine, Guangzhou, People's Republic of China; ${ }^{b}$ Department of Radiation Oncology, University of Michigan, Ann Arbor, Michigan, USA; 'Department of Medical Oncology, Sun Yat-sen University Cancer Center, State Key Laboratory of Oncology in South China, Collaborative Innovation Center for Cancer Medicine, Guangzhou, People's Republic of China; 'Imaging Diagnosis and Interventional Center, Sun Yat-sen University Cancer Center, State Key Laboratory of Oncology in South China, Collaborative Innovation Center for Cancer Medicine, Guangzhou, People's Republic of China; 'Department of Medical Statistics and Epidemiology, School of Public Health, Sun Yatsen University, Guangzhou, People's Republic of China.

*These authors contributed equally.

Submitted December 12, 2016; accepted for publication February 28, 2017.

The authors have disclosed that they have no financial interests, arrangements, affiliations, or commercial interests with the manufacturers of any products discussed in this article or their competitors. This work was supported by grants from National Natural Science Foundation of China (No.81572962); Health \& Medical Collaborative Innovation Project of
Guangzhou City, China (No. 201400000001); National Science \& Technology Pillar Program during the Twelfth Five-year Plan Period (2014BAI09B10); the Sun Yat-sen University Cultivating Foundation (16ykpy29); and the Planned Science and Technology Project of Guangdong Province (No. 2013B021800175).

Author contributions: Study conception and design: Tang, Y.P. Chen, Ma. Data acquisition: Tang, Y.P. Chen, Mao, Guo, L. Chen, Tian, Li. Data analysis and interpretation: Tang, Y.P. Chen, Wang, Lin, Ma. Access to raw data: Tang, Sung, Ma. Table and figure creation: Tang, Y.P. Chen, Wang. Drafting of manuscript: All authors. All authors had the opportunity to review the plan and outcome of analysis, participated in the preparation of this article, and provided final approval.

Correspondence: Jun Ma, MD, Department of Radiation Oncology, Sun Yat-sen University Cancer Center, State Key Laboratory of Oncology in South China, Collaborative Innovation Center of Cancer Medicine, 651 Dongfeng Road East, Guangzhou 510060, People's Republic of China. E-mail: majun2@mail.sysu.edu.cn 
Tang et al

\section{Background}

An accurate TNM staging system is crucial for evaluating the prognosis of patients with cancer, as well as treatment planning, treatment stratification, and coordinating clinical studies at different treatment centers. In response to newly acquired clinical data, timely modification of the TNM staging system is crucial to make it clinically useful and relevant worldwide. ${ }^{1}$ Thus, the AJCC and the Union for International Cancer Control (UICC) periodically modify the TNM staging system to reflect new developments in diagnostic and therapeutic techniques. Recently, the UICC/AJCC released the 8th edition of the staging system to further help physicians assign the proper treatment for individual patients and evaluate the results of management and clinical trials. ${ }^{2}$

Despite originating from similar cell or tissue lineages, the natural behavior of and therapeutic considerations for nasopharyngeal carcinoma (NPC) are distinct from other epithelial head and neck tumors. Recent advances in imaging modalities, such as MRI and PET scanning, have made it possible to measure tumor volume and extension more precisely in NPC. Additionally, developments in radiation technology, such as intensity-modulated radiotherapy (IMRT), have improved tumor target conformity and allow for safer dose escalations to the gross tumor volume (GTV). Although locoregional control has improved, distant metastasis remains the major failure pattern for NPC. The most recently published 8th edition of the UICC/AJCC for NPC was mainly derived from a large study in the IMRT era, in an attempt to maintain relevance with current management approaches and incorporate up-to-date clinical data. ${ }^{2,3}$

Table 1 compares the classification criteria and stage groupings of the 7 th and 8th editions of the UICC/AJCC staging system. One revision was to alter medial and lateral pterygoid muscle involvement from T4 to T2 and add prevertebral muscle involvement as T2. Another change was to replace the supraclavicular fossa (SCF) with the lower neck, and merge N3a and N3b into N3; T4 and N3 were also merged into stage IVA. These revisions are based on the use of IMRT and are supposed to optimize clinical practicability.

To validate the applicability of the 8 th edition of the UICC/AJCC staging system and provide further information for future refinements, we performed a large-scale retrospective in-depth comparison of the 7th and 8th editions in patients treated with IMRT in an area where NPC is endemic.

\section{Methods}

\section{Patient Characteristics}

A total of 1,790 consecutive patients with newly diagnosed, non-distant metastatic, histologically proven NPC treated with IMRT at our institution between November 2009 and February 2012 were retrospectively reviewed; those with a prior history of cancer were excluded. Of the 1,790 patients, 99.4\% had nonkeratinizing carcinoma and $0.6 \%$ had keratinizing squamous cell carcinoma according to the WHO classification. Median age was 45 years (range, $14-78$ years); 1,336 patients $(74.6 \%)$ were male and 454 (25.4\%) were female.

All patients completed a pretreatment evaluation, including a complete patient history, physical examination, hematology and biochemistry profiles, neck and nasopharyngeal MRI, chest radiography, abdominal sonography, and single-photon emission CT wholebody bone scan. Additionally, 29.1\% (520/1,790) of patients underwent an ${ }^{18} \mathrm{~F}$-fluorodeoxyglucose $(18 \mathrm{~F}$ FDG) PET/CT examination. The clinicopathological characteristics of the 1,790 patients are summarized in Table 2. All patients were restaged according to the 7th and 8th editions of the UICC/AJCC staging system. This study was reviewed and approved by the Institutional Review Board at Sun Yat-sen University Cancer Center.

\section{Treatment}

The nasopharyngeal and neck tumors of all patients were treated using radical radiotherapy based on IMRT for the entire course. Target volumes were delineated slice-by-slice on treatment planning CT scans using an individualized delineation protocol that complies with International Commission on Radiation Units and Measurements Reports 50 and 62. The prescribed doses were 66 to 72 Gy at 2.12 to 2.43 Gy/fraction to the planning target volume (PTV) of the primary GTV (GTVnx), 64 to 70 Gy per 28 to 33 fractions to the PTV of the GTV of involved lymph nodes (GTVnd), 60 to 63 Gy per 28 to 33 fractions to the PTV of the high-risk clinical target volume (CTV1), and 54 to 56 Gy per 28 to 33 fractions to the PTV of the low-risk clinical target volume (CTV2). Median dose delivered was $72.77 \pm 1.26$ Gy to the PTV of the 
Validation of the 8th NPC Staging System

\begin{tabular}{|c|c|c|}
\hline & 7th Edition & 8th Edition \\
\hline \multicolumn{3}{|l|}{ T category } \\
\hline & $\begin{array}{l}\text { T1: Nasopharynx, oropharynx, or nasal cavity without } \\
\text { parapharyngeal extension }\end{array}$ & $\begin{array}{l}\text { T1: Nasopharynx, oropharynx, or nasal cavity without parapharyngeal } \\
\text { extension }\end{array}$ \\
\hline & T2: Parapharyngeal extension & $\begin{array}{l}\text { T2: Parapharyngeal extension, adjacent soft tissue involvement (medial } \\
\text { pterygoid, lateral pterygoid, prevertebral muscles) }\end{array}$ \\
\hline & T3: Bony structures of skull base and/or paranasal sinuses & $\begin{array}{l}\text { T3: Bony structures (skull base, cervical vertebra) and/or paranasal } \\
\text { sinuses }\end{array}$ \\
\hline & $\begin{array}{l}\text { T4: Intracranial, cranial nerves, hypopharynx, orbit, } \\
\text { infratemporal fossa/masticator space }\end{array}$ & $\begin{array}{l}\text { T4: Intracranial extension, cranial nerve, hypopharynx, orbit, extensive } \\
\text { soft tissue involvement (beyond the lateral surface of the lateral } \\
\text { pterygoid muscle, parotid gland) }\end{array}$ \\
\hline \multicolumn{3}{|l|}{$\mathrm{N}$ category } \\
\hline & N0: No regional lymph node metastasis & N0: No regional lymph node metastasis \\
\hline & $\begin{array}{l}\text { N1: Unilateral cervical, unilateral or bilateral } \\
\text { retropharyngeal lymph nodes above the supraclavicular } \\
\text { fossa; } \leq 6 \mathrm{~cm}\end{array}$ & $\begin{array}{l}\text { N1: Retropharyngeal (regardless of laterality) } \\
\text { Cervical: unilateral, } \leq 6 \mathrm{~cm} \text {, and above caudal border of cricoid cartilage }\end{array}$ \\
\hline & $\begin{array}{l}\text { N2: Bilateral metastasis in lymph nodes, } \leq 6 \mathrm{~cm} \text { in greatest } \\
\text { dimension, above the supraclavicular fossa }\end{array}$ & $\begin{array}{l}\text { N2: Cervical: bilateral, } \leq 6 \mathrm{~cm} \text {, and above caudal border of cricoid } \\
\text { cartilage }\end{array}$ \\
\hline & N3a: $>6 \mathrm{~cm}$ in dimension & $\begin{array}{l}\text { N3: }>6 \mathrm{~cm} \text { and/or below caudal border of cricoid cartilage } \\
\text { (regardless of laterality) }\end{array}$ \\
\hline & N3b: Supraclavicular fossa & \\
\hline \multicolumn{3}{|l|}{ Stage/Group } \\
\hline & I: T1 No Mo & I: T1 No Mo \\
\hline & II: T2 N0-1 M0, T1 N1 Mo & II: T2 N0-1 M0, T1 N1 Mo \\
\hline & III: T1-3 N2 M0, T3 N0-1 M0 & III: T3 N0-2 M0, T1-2 N2 M0 \\
\hline & IVA: T4 N0-2 MO & IVa: T4 or N3 M0 \\
\hline & IVB: Any T N3 M0 & IVb: Any T, any N M1 \\
\hline & IVC: Any T, any N M1 & \\
\hline
\end{tabular}

Abbreviations: UICC/AJCC, Union for International Cancer Control/American Joint Committee on Cancer.

GTVnx. All targets were treated simultaneously using the simultaneous integrated boost technique. During the study, institutional guidelines recommended only IMRT for stage I NPC and concurrent chemoradiotherapy with or without neoadjuvant/adjuvant chemotherapy for stage II to IVB NPC (according to the 7 th edition of the UICC/AJCC). In this cohort, 1,250 of 1,329 patients $(94.1 \%)$ with locoregionally advanced NPC (stage III to IVB) received chemotherapy. When possible, salvage treatments (intracavitary brachytherapy, surgery, or chemotherapy) were provided for patients with documented relapse or persistent disease.

\section{Follow-Up}

Patient follow-up was measured from the first day of therapy to the day of last examination or death. Patients were examined at least every 3 months during the first 2 years, with follow-up examinations every 6 months for 3 years thereafter or until death. Median follow-up was 57.4 months (range, 1.3-78.5 months).

The primary end point for this analysis was disease-free survival (DFS; time to failure or death from any cause, whichever occurred first). Secondary end points included overall survival (OS; time to death from any cause), locoregional failure-free survival (LRFFS; time to locoregional persistence/ recurrence), and distant failure-free survival (DFFS; time to distant metastasis). Patients with residual or recurrent local disease underwent biopsy to confirm malignancy. Additional tests were ordered when indicated to detect local or distant failure.

\section{Statistical Analysis}

All analyses were performed using SPSS version 19.0 (IBM Corporation, Armonk, NY) and R version 
Tang et al

\begin{tabular}{|c|c|}
\hline Characteristic & N (\%) \\
\hline \multicolumn{2}{|l|}{ Sex } \\
\hline Male & $1,336(74.6 \%)$ \\
\hline Female & $454(25.4 \%)$ \\
\hline \multicolumn{2}{|l|}{ Age, y } \\
\hline$\leq 45$ & $946(52.8 \%)$ \\
\hline$>45$ & $844(47.2 \%)$ \\
\hline \multicolumn{2}{|l|}{ Histologic type } \\
\hline Keratinizing squamous cell carcinoma & $10(0.6 \%)$ \\
\hline Nonkeratinizing carcinoma & $1,780(99.4 \%)$ \\
\hline \multicolumn{2}{|l|}{ Chemotherapy } \\
\hline Induction + concurrent & $667(37.3 \%)$ \\
\hline Concurrent only & $652(36.4 \%)$ \\
\hline Concurrent + adjuvant & $23(1.3 \%)$ \\
\hline No chemotherapy & $251(14.0 \%)$ \\
\hline
\end{tabular}

3.0.0 (www.r-project.org). Actuarial rates were estimated using the Kaplan-Meier method; survival curves were compared using the log-rank test. ${ }^{4}$

Multivariate analyses with the Cox proportional hazards model were used to test for independent significance by backward elimination of insignificant explanatory variables. ${ }^{5}$ Cox proportional hazards model was also used to calculate hazard ratios (HR). Host factors (age and sex) and treatment (chemotherapy) were included as covariates in all tests. The performance of each staging system was compared using the Akaike information criterion (AIC) ${ }^{6}$ and Harrell's concordance index (c-index). ${ }^{7}$ Both AIC and c-index values were calculated using the Cox proportional hazards model. The AIC refers to information loss when predicting outcome in a model; an optimal model gives the lowest AIC value. Harrell's c-index measures the ability to predict outcomes; a higher c-index indicates a better efficacy to discriminate outcomes. Two-tailed $P$ values $<.05$ were considered statistically significant.

\section{Results}

\section{T Category Classification}

The distributions of the $\mathrm{T}$ category classifications defined by the 7 th and 8th editions of the UICC/ AJCC staging system for the study population are shown in supplemental eTable 1, available with this article at JNCCN.org. Of the 541 patients with T4 disease according to the 7th edition, $17(0.9 \%)$ and $188(10.5 \%)$ were downgraded to T2 and T3 in the 8th edition, because they had medial or lateral pterygoid muscle involvement, respectively; 12 patients $(0.7 \%)$ with T1 NPC in the 7th edition were classified as T2 in the 8th edition, because they had prevertebral muscle involvement.

The 5-year DFS, OS, LRFFS, and DFFS rates for each $\mathrm{T}$ category of the 7 th and 8 th editions are presented in Table 3, and Figure 1 shows the LRFFS and DFS curves for the T categories of the 7 th and 8th editions. With the improvement in locoregional control provided by IMRT, LRFFS was generally high for all $\mathrm{T}$ categories of both editions (Figure $1 \mathrm{~A}$ and B). The 5-year DFS rates for T1-T4 NPC were $88 \%$, $81 \%, 82 \%$, and $73 \%$, respectively, in the 7 th edition and $88 \%, 82 \%, 81 \%$, and $70 \%$ in the 8 th edition (Table 3). Although the 8th edition distinguishes T4 NPC more accurately, it is still not completely satisfactory. In both editions, the LRFFS and DFS curves for T2 and T3 almost overlapped, without significant differences (Figure 1C and D). Thus, it seems reasonable to merge T2 and T3 in future editions. Probably due to the difficultly of differentiating $\mathrm{T} 2$ and $\mathrm{T} 3$, the AIC and c-index values for all end points were similar between the 7th and 8th editions (Table 3).

\section{N Category Classification}

Distribution of $\mathrm{N}$ category classifications as defined by the 7 th and 8th editions are shown in supplemental eTable 2. Replacing the N3b criterion of the SCF with the lower neck (below the caudal border of cricoid cartilage) led to upstaging of 73 patients (4.1\%) from N1-2 to N3.

Table 3 presents the 5 -year survival rates for different end points of the $\mathrm{N}$ categories defined by each staging system. Figure 2 shows the DFFS and DFS curves for each $\mathrm{T}$ category as defined by the 7 th and 8th editions. In the 8th edition, the differences between each $\mathrm{N}$ category reached statistical significance, indicating that the 8 th edition has a better prognostic value with respect to $\mathrm{N}$ category classification than the 7th edition. Thus, it is not surprising that the 8 th edition had higher AIC and c-index values for all end points compared with the 7 th edition (Table 3 ).

\section{Stage Group Classification}

Distribution of stage groups for the 7 th and 8 th editions are shown in supplemental eTable 3. In the 8th 
Validation of the 8th NPC Staging System

\begin{tabular}{|c|c|c|c|c|c|c|c|c|c|}
\hline \multicolumn{5}{|c|}{ 7th Edition } & \multicolumn{5}{|c|}{ 8th Edition } \\
\hline T Category & DFS & os & LRFFS & DFFS & T Category & DFS & os & LRFFS & DFFS \\
\hline T1 & $88 \%$ & $94 \%$ & $94 \%$ & $93 \%$ & $\mathrm{~T} 1$ & $88 \%$ & $94 \%$ & $94 \%$ & $93 \%$ \\
\hline $\mathrm{T} 2$ & $81 \%$ & $88 \%$ & $89 \%$ & $91 \%$ & $\mathrm{~T} 2$ & $82 \%$ & $89 \%$ & $90 \%$ & $91 \%$ \\
\hline T3 & $82 \%$ & $90 \%$ & $91 \%$ & $90 \%$ & T3 & $81 \%$ & $88 \%$ & $90 \%$ & $89 \%$ \\
\hline T4 & $73 \%$ & $80 \%$ & $88 \%$ & $84 \%$ & T4 & $70 \%$ & $77 \%$ & $87 \%$ & $81 \%$ \\
\hline $\mathrm{AlC}^{\mathrm{a}}$ & 5042.9 & 3197.0 & 2870.4 & 2350.9 & & 5042.4 & 3198.3 & 2868.9 & 2350.0 \\
\hline c-index ${ }^{a}$ & 0.60 & 0.65 & 0.60 & 0.59 & & 0.59 & 0.64 & 0.60 & 0.60 \\
\hline N Category & DFS & os & LRFFS & DFFS & N Category & DFS & os & LRFFS & DFFS \\
\hline No & $91 \%$ & $94 \%$ & $95 \%$ & $95 \%$ & No & $91 \%$ & $94 \%$ & $95 \%$ & $95 \%$ \\
\hline N1 & $81 \%$ & $88 \%$ & $90 \%$ & $90 \%$ & N1 & $82 \%$ & $90 \%$ & $90 \%$ & $91 \%$ \\
\hline N2 & $72 \%$ & $81 \%$ & $88 \%$ & $81 \%$ & $\mathrm{~N} 2$ & $72 \%$ & $81 \%$ & $88 \%$ & $81 \%$ \\
\hline N3a & $63 \%$ & $64 \%$ & $89 \%$ & $66 \%$ & N3 & $59 \%$ & $67 \%$ & $86 \%$ & $69 \%$ \\
\hline N3b & $55 \%$ & $68 \%$ & $83 \%$ & $71 \%$ & & & & & \\
\hline $\mathrm{AlCa}$ & 5011.0 & 3184.8 & 2840.9 & 2349.6 & $\mathrm{AlC}^{\mathrm{a}}$ & 4990.1 & 3157.4 & 2813.7 & 2346.2 \\
\hline c-index ${ }^{a}$ & 0.62 & 0.66 & 0.65 & 0.58 & c-index ${ }^{a}$ & 0.64 & 0.69 & 0.68 & 0.59 \\
\hline Stage/ Group & DFS & OS & LRFFS & DFFS & Stage/ Group & DFS & os & LRFFS & DFFS \\
\hline I & $98 \%$ & $100 \%$ & $99 \%$ & $99 \%$ & 1 & $98 \%$ & $100 \%$ & $99 \%$ & $99 \%$ \\
\hline II & $86 \%$ & $93 \%$ & $91 \%$ & $94 \%$ & II & $86 \%$ & $93 \%$ & $92 \%$ & $93 \%$ \\
\hline III & $83 \%$ & $91 \%$ & $91 \%$ & $91 \%$ & III & $82 \%$ & $90 \%$ & $91 \%$ & $91 \%$ \\
\hline IVa & $73 \%$ & $80 \%$ & $88 \%$ & $84 \%$ & IVa & $68 \%$ & $75 \%$ & $86 \%$ & $79 \%$ \\
\hline $\mathrm{IVb}$ & $59 \%$ & $67 \%$ & $86 \%$ & $69 \%$ & & & & & \\
\hline $\mathrm{AlC}^{\mathrm{a}}$ & 4995.2 & 3148.1 & 2832.5 & 2344.7 & $\mathrm{AlC}^{\mathrm{a}}$ & 4995.6 & 3149.0 & 2832.7 & 2341.7 \\
\hline c-index ${ }^{a}$ & 0.64 & 0.70 & 0.66 & 0.60 & c-index ${ }^{a}$ & 0.63 & 0.70 & 0.66 & 0.60 \\
\hline
\end{tabular}

Abbreviations: AIC, Akaike information criterion; c-index, Harrell's concordance index; DFFS, distant failure-free survival; DFS, disease-free survival; LRFFS, locoregional failure-free survival; OS, overall survival; UICC/AJCC, Union for International Cancer Control/American Joint Committee on Cancer.

aThe AIC and c-index values were calculated using a Cox proportional hazards regression model that included age ( $\leq 45$ vs $>45$ years), sex (male vs female), and chemotherapy (yes vs no).

edition, 184 patients (10.2\%) with stage IVA disease in the 7 th edition were downstaged to stage II or III.

The 5-year DFS, OS, LRFFS, and DFFS rates for the different stage groups are shown in Table 3; Figure 3 shows the OS and DFS curves for the stage groups. Similarly, the OS and DFS curves for stage II and III were not clearly separated in either edition (Figure 3), indicating that the 8th edition does not distinguish T2 and T3 disease better than the 7th edition. The 7th and 8th editions had similar AIC and c-index for all end points.

\section{Discussion}

The UICC/AJCC TNM staging system is essential for predicting prognosis, guiding treatment strategies for different risk groups, and facilitating the exchange of information between oncology centers. The extent of local invasion, regional lymphatic spread, and distant metastasis, as reflected by the TNM staging system, are the most important prognostic factors in NPC. This is the first comparison of the 7th and 8th editions of the UICC/AJCC TNM staging system for NPC, and analysis indicates that the 8th edition has improved prognostic value, especially with respect to $\mathrm{N}$ category classification. However, the current 8 th edition is not completely satisfactory, with a lack of separation between T2 and T3 NPC and stage II and III NPC.

The SCF, a triangular region defined by Ho, is constituted by 3 clinical landmarks ${ }^{1}$ that are difficult to describe on cross-sectional imaging and is used in the 7 th edition. $\mathrm{Ng}$ et $\mathrm{al}^{8}$ first used the radiologic nodal levels IV and Vb, as defined by Som et al, ${ }^{9}$ to replace Ho's triangle, and found this substitution useful. Pan et $\mathrm{al}^{3}$ also reported that it was feasible to use 
Tang et al

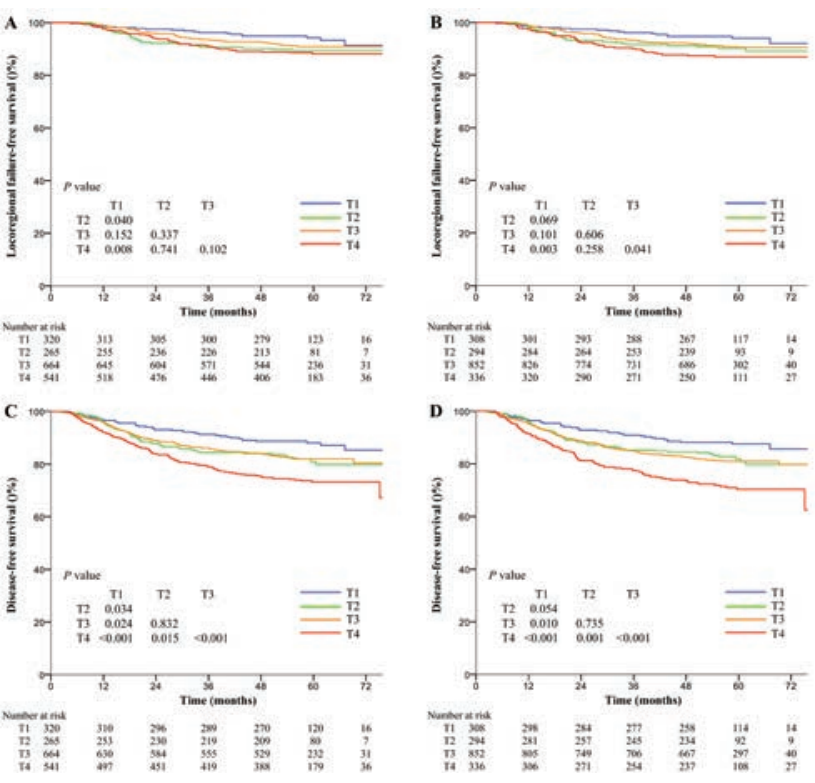

Figure 1. Locoregional failure-free survival (A, B) and disease-free survival $(C, D)$ for each $T$ category as defined by the 7 th $(A, C)$ and 8 th $(B, D)$ editions of the Union for Internation Cancer Control/AJCC staging system.
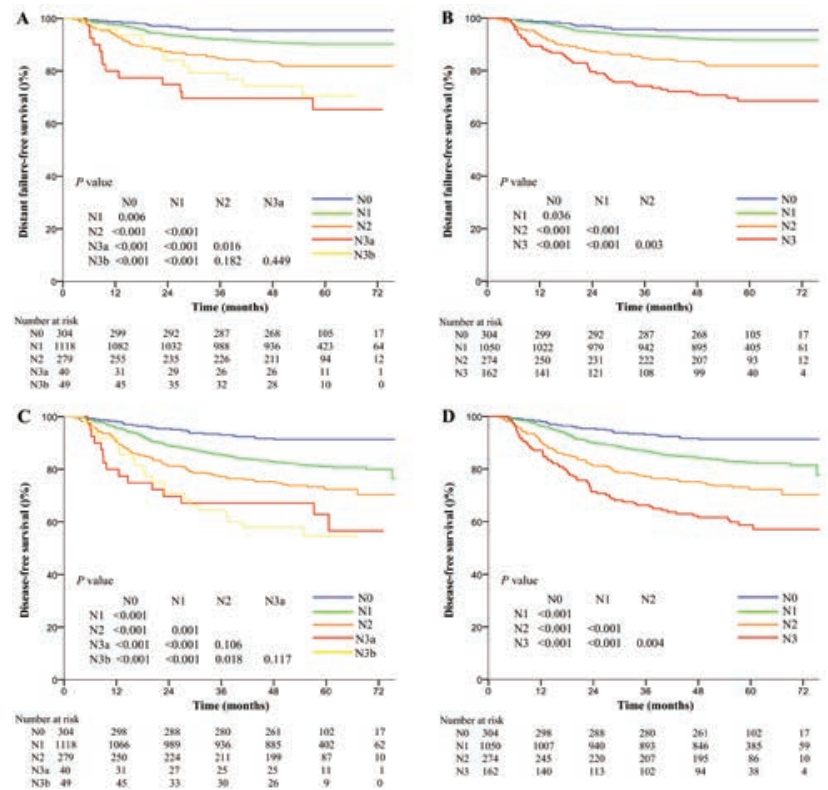

Figure 2. Distant failure-free survival $(A, B)$ and disease-free surviva $(C, D)$ for each $N$ category as defined by the 7 th $(A, C)$ and 8 th $(B, D)$ editions of the Union for International Cancer Control/AJCC staging system. levels IV and Vb and the SCF to replace Ho's triangle. Therefore, in the 8th edition, the SCF was replaced with the lower neck region (below the caudal border of the cricoid cartilage). Moreover, as distant metastasis-free survival and DFS did not differ significantly between $\mathrm{N} 3 \mathrm{a}$ and $\mathrm{N} 3 \mathrm{~b}$ of the 7 th edition, these categories were merged into $\mathrm{N} 3$ in the 8th edition. Accordingly, the survival curves for different $\mathrm{N}$ categories were well-separated in the 8 th edition, and the prognostic model predicted outcomes fairly well.

Based on previous research, medial and lateral pterygoid muscle involvement was downgraded from T4 to T2, and prevertebral muscle involvement was added as T2..$^{3,10-12}$ However, this study proves the 8 th edition still results in a lack of separation in local control between T2 and T3. The recent study by Pan et $\mathrm{al}^{3}$ that proposed the 8th edition also found no significant differences in local failure between $\mathrm{T} 2$ and T3, only a significant difference in OS (and a trend toward a difference in distant failure). Generally, advanced $\mathrm{T}$ category is associated with poor locoregional control and poor OS; advanced $\mathrm{N}$ category has an increased risk of distant failure and death. Lai et $\mathrm{al}^{13}$ found that the improved treatment outcomes of IMRT (2-dimensional RT) were primarily due to a higher local tumor control rate, confirming that distant metastasis is now the predominant mode of treatment failure in NPC. Therefore, the prognostic value of $\mathrm{T}$ category may have become weaker than that of $\mathrm{N}$ due to the excellent
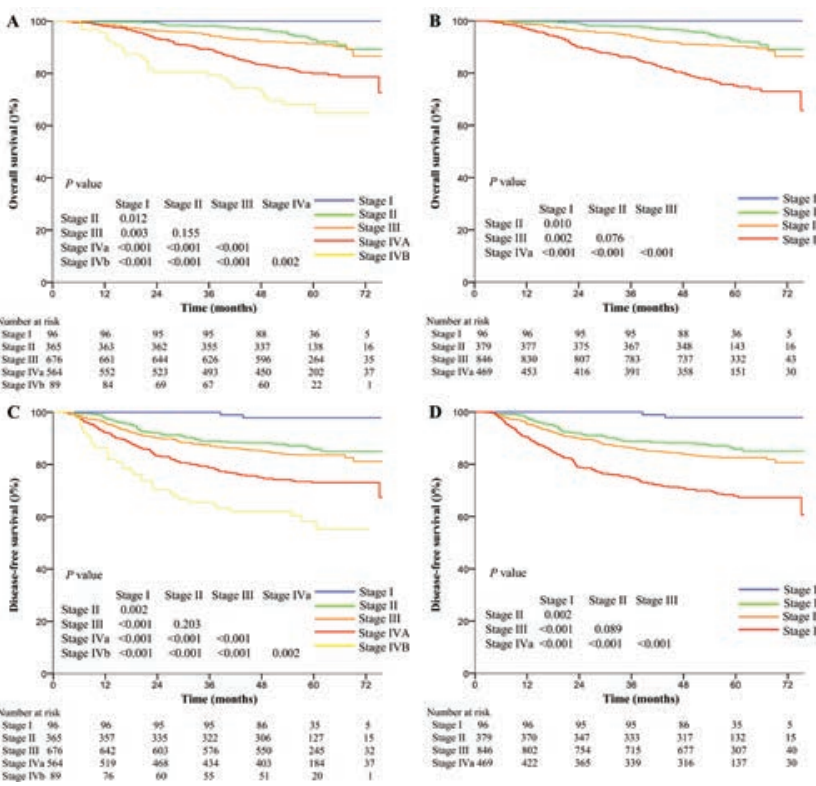

Figure 3. Overall survival (A, B) and disease-free survival (C, D) for each stage group as defined by the 7th $(A, C)$ and 8th $(B, D)$ editions of the Union for International Cancer Control/AJCC staging system. 
rate of local control. It seems reasonable to suggest $\mathrm{T}$ category classification could be further optimized, for example through merging $\mathrm{T} 2$ and $\mathrm{T} 3$ or incorporating other distinguishing factors.

Besides optimizing the basic TNM staging system, further refinement by incorporating relevant prognostic factors will be useful for guiding individualized treatment strategies. The 8th edition only incorporates parameters describing the anatomic extent of the tumor, as determined by clinical and pathologic methods. The AJCC has increasingly recognized the growing need for more accurate and probabilistic individualized outcome prediction models-incorporating additional anatomic and nonanatomic prognostic factors beyond the TNM system-to develop a precision medicine approach. Additional relevant prognostic factors, such as Epstein-Barr virus DNA load, tumor volume, and other nonanatomic factors, should be considered and potentially combined with the TNM staging system in future revisions. ${ }^{14}$

One limitation of the current study is that it retrospectively enrolled patients at a single center in an endemic area, although this was a large-scale study. Multi-institutional studies are necessary to confirm the applicability of the 8th edition and our proposed modifications to this staging system. Another limitation is that PET/CT was only performed on approximately $30 \%$ of patients in this series, and with its well-recognized superiority for detecting metastases, there is little doubt that PET/CT may improve staging accuracy. ${ }^{15,16}$ Finally, although all patients were treated with IMRT, which is the current standard of care, it is not available everywhere. The proposed refinements may not be applicable in institutions where IMRT is not available or feasible.

\section{Conclusions}

The 8th edition of the UICC/AJCC staging system for NPC enables a more accurate prediction of treatment outcomes than the 7 th edition. However, the limitations discussed in this study need to be addressed in future revisions of this staging system. Because local control has improved in the modern era, it seems reasonable that T-category classification should be further optimized. Additional studies are warranted to further validate the 8 th edition in other cohorts of patients.

\section{References}

1. Edge SB, Byrd DR, Compton CC, et al, eds. AJCC Cancer Staging Manual, 7th ed. New York, NY: Springer; 2010.

2. Amin MB, Edge S, Greene F, et al, eds. AJCC Cancer Staging Manual, 8th ed. New York, NY: Springer; 2016.

3. Pan JJ, Ng WT, Zong JF, et al. Proposal for the 8th edition of the AJCC/ UICC staging system for nasopharyngeal cancer in the era of intensitymodulated radiotherapy. Cancer 2016;122:546-558.

4. Kaplan EL, Meier P. Nonparameric estimation from incomplete observation. J Am Stat Assoc 1958;53:457-481.

5. Cox DR. Regression models and life tables. J R Stat Soc Series B Stat Methodol 1972;34:187-220

6. Akaike H. A new look at the statistical model identification. IEEE Trans Automat Contr 1974;19:716-723.

7. Harrell FE Jr, Califf RM, Pryor DB, et al. Evaluating the yield of medical tests. JAMA 1982;247:2543-2546.

8. Ng WT, Lee AW, Kan WK, et al. N-staging by magnetic resonance imaging for patients with nasopharyngeal carcinoma: pattern of nodal involvement by radiological levels. Radiother Oncol 2007;82:70-75.

9. Som PM, Curtin HD, Mancuso AA. Imaging-based nodal classification for evaluation of neck metastatic adenopathy. AJR Am J Roentgenol 2000;174:837-844.

10. Sze H, Chan LL, Ng WT, et al. Should all nasopharyngeal carcinoma with masticator space involvement be staged as T4? Oral Oncol 2014;50:11881195.

11. Xiao Y, Pan J, Chen Y, et al. Prognostic value of MRI-derived masticator space involvement in IMRT-treated nasopharyngeal carcinoma patients. Radiat Oncol 2015;10:204.

12. Zhang GY, Huang Y, Cai XY, et al. Prognostic value of grading masticator space involvement in nasopharyngeal carcinoma according to MR imaging findings. Radiology 2014;273:136-143.

13. Lai SZ, Li WF, Chen L, et al. How does intensity-modulated radiotherapy versus conventional two-dimensional radiotherapy influence the treatment results in nasopharyngeal carcinoma patients? Int J Radiat Oncol Biol Phys 2011;80:661-668.

14. Chan KC. Plasma Epstein-Barr virus DNA as a biomarker for nasopharyngeal carcinoma. Chin J Cancer 2014;33:598-603.

15. Ng SH, Chan SC, Yen TC, et al. Staging of untreated nasopharyngeal carcinoma with PET/CT: comparison with conventional imaging work-up. Eur J Nucl Med Mol Imaging 2009;36:12-22.

16. Yen TC, Chang JT, Ng SH, et al. The value of $18 \mathrm{~F}$-FDG PET in the detection of stage MO carcinoma of the nasopharynx. J Nucl Med 2005;46:405-410. 